\section{Considerations Concerning the Numerical Computation of Mixing Times in Steelmaking Ladles}

\section{Introduction}

Liquid mixing phenomena in gas stirred metallurgical reactors (e.g., furnaces and ladles) have been the subject of wide spread interest in recent years. ${ }^{1-9)}$ A large number of investigations, both experimental ${ }^{1-6)}$ as well as theoretical ${ }^{7-9}$ have been carried out. In a recent contribution, Turkoglu and Farouk ${ }^{10)}$ described a purely computational study concerning liquid mixing phenomena in steelmaking ladles. The authors ${ }^{10)}$ made use of the commercial code PHOENICS, ${ }^{11)}$ and proposed that a rigorous two fluid hydrodynamic model be used for fluid flow calculations, in conjunction with the standard $k-\varepsilon$ mode $^{12)}$ for simulating turbulence phenomena. Coupling these with an appropriate species conservation model for predicting $95 \%$ bulk mixing times ${ }^{9)}$ in cylindrical steelmaking ladles, the authors made a computational investigation of the influence of a wide range of operating conditions on mixing times. Given an absence of any physically equivalent measurements for high temperature gas stirred systems, these authors assessed the appropriateness of their numerically computed results against those predicted on the basis of an empirically derived correlation proposed earlier by Mazumdar and Guthrie. ${ }^{9)}$ The comparison, as presented by Turkoglu and Farouk, is reproduced from Ref. 10) as Fig. 1 in

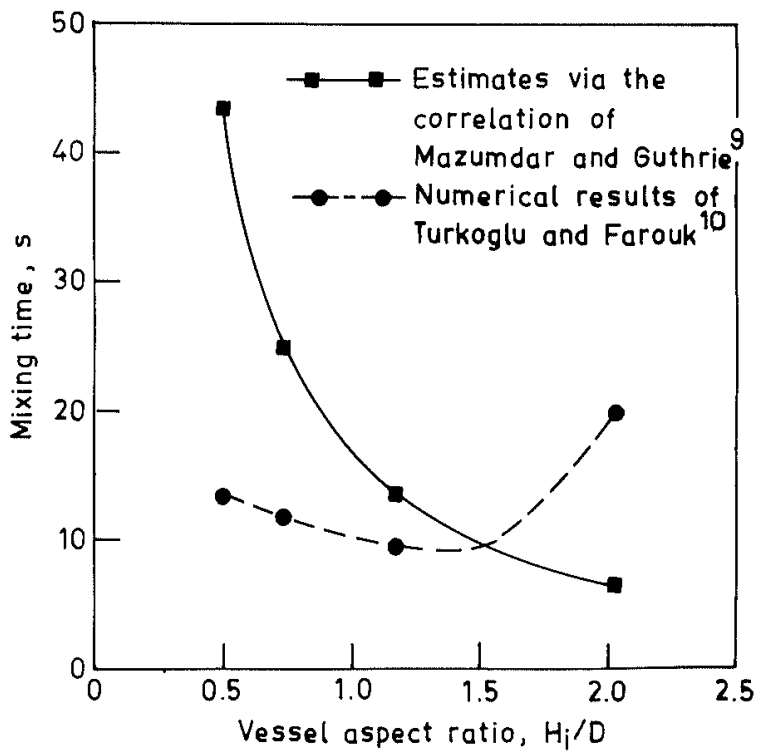

Fig. 1. Numerical estimates of mixing times as a function of vessel aspect ratio and their comparison with those deduced on the basis of Eq. (4) [reproduced from Ref. 10)]. this article.

In explaining the differences between the two sets of estimates of mixing times $v s$, vessel aspect ratio (as illustrated in Fig. 1), Turkoglu and Farouk considered their numerical estimates to be more realistic than those calculated from the correlation proposed by Mazumdar and Guthrie. In justifying their work, arguments and analyses contradictory to observations documented in the literature are made. The purpose of the present work is to asses their work, ${ }^{10}$ ) to reveal inherent ambiguities, and to present a more coherent analysis of mixing time calculations/measurements in gas stirred ladle systems. To reach these objectives, it is appropriate to briefly reiterate salient features of our earlier work ${ }^{9)}$ as well as other relevant literature, and re-examine some of the results, arguments and analyses presented by Turkoglu and Farouk. ${ }^{10)}$

\section{The Mixing Time Correlation of Mazumdar and Guthrie}

Mazumdar and Guthrie ${ }^{9)}$ presented an elaborate theoretical treatment on the subject of mixing phenomena in gas stirred melts and suggested that for the two possible limiting situations of purely convective and eddy diffusive transport controlled mixing, mixing times for tracers added to gas agitated refining vessels can be represented as:

$$
\tau_{m}=C_{1} R^{5 / 3} /\left[(\beta Q)^{1 / 3} \cdot L^{1 / 4}\right]
$$

for dispersion dominated by bulk circulation phenomena and,

$$
\tau_{m}=C_{2} R^{7 / 3} /\left[(\beta Q)^{1 / 3} \cdot L\right]
$$

for dispersion dominated by turbulent diffusion phenomena.

Implicit in Eqs. (1) and (2) are expressions for the average speed of liquid recirculation ${ }^{13)}$ and the bulk average effective viscosity ${ }^{14)}$ proposed by Sahai and Guthrie. These as well as details underlying the derivation of Eqs. (1) and (2) are discussed at length in Ref. 9).

Mazumdar and Guthrie also carried out a large number of measurements in air water systems and concurrently provided numerically computed mixing times. The theoretical analysis as well as experimental measurements demonstrated explicitly, that liquid mixing phenomena in gas stirred ladle systems cannot be characterised solely in terms of eddy diffusion nor in terms of convective transport mechanisms (viz., Eqs. (1) and (2)). Furthermore the authors argued from both a theoretical and experimental standpoint that Eqs. (1) and (2) (which were essentially derived from a procedure suggested by Asai and coworkers ${ }^{1)}$ ) are not at all representative of either convection or eddy diffusion 
controlled mixing in ladles. It was, however, noted ${ }^{9)}$ that experimentally measured mixing times can be described reasonably via a functional relationship of the type expressed by Eq. (2)., viz.,

$$
\tau_{m} \propto R^{7 / 3} /\left[(\beta Q)^{1 / 3} \cdot L\right]
$$

As outlined in Ref. 9), the constant of proportionality in Eq. (3) was deduced from experimental measurements of mixing times in air-water systems and was found to be about $25.4\left(\mathrm{~m}^{-1} \mathrm{~s}^{2}\right)^{1 / 3}$. It is again emphasized that Eq. (3) describes mixing in gas stirred ladle systems purely from a experimental point of view and as such, has no theoretical basis what so ever. Consequently, the fact that mixing times can be represented adequately via Eq. (3), which has an analogous form to Eq. (2) was considered to be purely fortuitous. ${ }^{9)}$ Thus, apparent similarities between Eqs. (2) and (3) did not imply that eddy diffusion phenomena dominates mixing processes in ladles.

The experimental configuration applied in our earlier studies $^{9)}$ essentially involved injection of gas through a central, vertically submerged, lance into a cylindrical tank containing water. Furthermore, since the kinetic energy of the incoming injected gas was practically negligible ( $<5 \%$ or so), the potential energy afforded by the rising bubbles was considered to be the only source of stirring energy in the gas stirred bath. On the basis of these, Eq. (3) can also be repesented as:

$$
\tau_{m}=37 \varepsilon_{m}^{-1 / 3} R^{5 / 3} L^{-1}
$$

in which, $\varepsilon_{m}$ is the rate of specific potential energy input to the gas stirred system $\left(=\rho_{L} g Q L \beta / \rho_{L} \pi R^{2} L\right)$. Equations (3) and (4) as discussed in Ref. 9), were specific to a set of operating conditions and therefore, are only strictly valid under the following conditions:

(i) gas stirred ladle systems $(0.5 \leq L / D \leq 2.0)$ under central gas injection conditions and for a degree of bulk mixing equal to $95 \%$,

(ii) negligible kinetic energy input to the system,

(iii) specific potential energy input rate in the order of $10^{-2} \mathrm{~W} / \mathrm{kg}$ (e.g., corresponding to a maximum gas flow rate of about $100 \mathrm{l} / \mathrm{min}$ in a $1000 \mathrm{~kg}$ water model with $L / D=1.0$ ).

Given this background, it is now worthwhile taking a fresh look at the results and analysis presented by Turkoglu and Farouk.

\section{Analysis of Results Presented by Turkoglu and Farouk}

In order to explain the differences between the two sets of estimates, ${ }^{9,10)}$ Turkoglu and Farouk attributed the discrepancy to be inherent to the derivation of the mixing time correlation (viz., Eq. (4)). These authors ${ }^{10)}$ went on to suggest that in deriving Eqs. (3) and (4), Mazumdar and Guthrie assumed uniform turbulent/eddy viscosity and uniform liquid recirculation velocity in the entire bath. This is clearly misleading since the previous work (the essence of which is summarised in the preceding
Table 1. Summary of experimental conditions applied in

\begin{tabular}{|c|c|c|}
\hline Parameters & $\begin{array}{l}\text { Investigation of } \\
\text { Turkoglu and } \\
\text { Farouk }^{10)}\end{array}$ & $\begin{array}{l}\text { Investigation of } \\
\text { Mazumdar and } \\
\text { Guthrie }\end{array}$ \\
\hline Liquid & Steel & Water \\
\hline Vessel size & 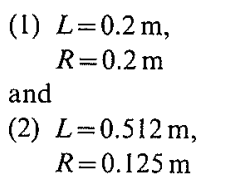 & $\begin{aligned} \text { (1) } L & =0.93 \mathrm{~m} \\
R & =0.56 \mathrm{~m} \\
\text { and } & \\
\text { (2) } L & =0.65 \mathrm{~m} \\
R & =0.305 \mathrm{~m}\end{aligned}$ \\
\hline Nozzle diameter $(\mathrm{m})$ & 0.002 & 0.006 \\
\hline Gas flow rate $\left(\mathrm{m}^{3} / \mathrm{s}\right)$ & $3.14 \times 10^{-3}$ & $6.8 \times 10^{-4}$ to $1.0 \times 10^{-3}$ \\
\hline Mass of fluid (kg) & 176 & 900 and 190 \\
\hline $\begin{array}{l}\text { Specific potential } \\
\text { energy input rate } \\
(\mathrm{W} / \mathrm{kg})\end{array}$ & 0.77 and 1.97 & 0.03 and 0.07 \\
\hline
\end{tabular}
the two investigations. ${ }^{9.10)}$

section), explicitly stated that the mixing time correlations as expressed by Eqs. (3) and (4) were purely empirical. Thus, the arguments Turkoglu and Farouk provide to explain the source of discrepancy between the two sets of work, ${ }^{9,10)}$ as reflected in Fig. 1, are erroneous and justify further analysis.

Thus, Turkoglu and Farouk simulated, mathematically, mixing times in ladles of various sizes containing about $176 \mathrm{~kg}$ of liquid steel at a gas flow rate of $3.142 \times 10^{-3} \mathrm{~m}^{3} / \mathrm{s}$ (approximately $200 \mathrm{l} / \mathrm{min}$ ). On the other hand, Mazumdar and Guthrie proposed their experimental correlation under conditions where a maximum of $100 \mathrm{l} / \mathrm{min}$ of gas flow rate was used to stir about $1000 \mathrm{~kg}$ of water contained in a water model ladle. Similarly, Turkoglu and Farouk applied the following expression to estimate the rate of potential energy input to the gas stirred system e.g.,

$$
W_{B}=m_{g} R_{g} T \ln \left[1+\left(H_{i} \rho_{L} g / P_{a}\right)\right]
$$

which produces significantly different estimates of equivalent energy input rate inferred from the formula applied by Mazumdar and Guthrie viz.,

$$
W_{B}=\rho_{L} g Q H_{i}
$$

It is important to recognise here that Eq. (6) is essentially the same as that proposed by Sano and Mori, ${ }^{14)}$ though derived from a completely different stand point. Thus, adopting Eq. (6) to either systems ${ }^{9,10)}$ (e.g., since $\left.P_{a} \gg \rho_{L} g H_{i}\right)^{*}$, it is readily seen (viz., Table 1) that the specific potential energy input rates considered by Turkoglu and Farouk are at least an order of magnitude greater than those for which Eqs. (3) and (4) were found to be applicable. A comparison between the experimental conditions applied in the two investigations $^{9,10)}$ are in Table 1.

Figure 2 presents a typical set of results reproduced from Ref. 10) which shows the consequences of higher rates of specific gas consumption or potential energy input on the overall hydrodynamics of the gas stirred

\footnotetext{
* A detailed consideration on the derivation of various formulations for potential energy input rate to the gas stirred system has been recently presented by Schwarz. ${ }^{15}$
} 


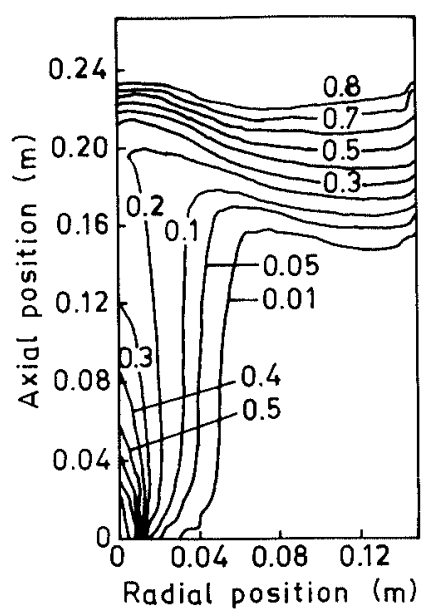

a.

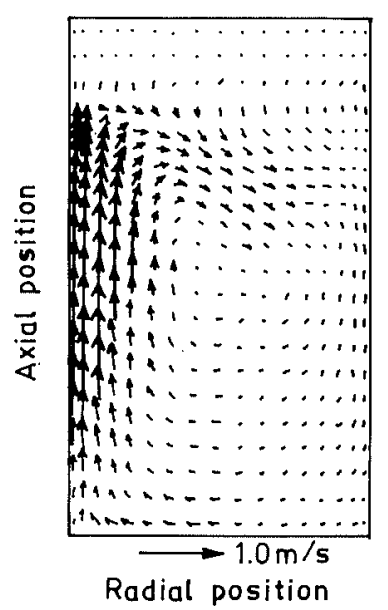

b.
Fig. 2. (a) Computed contour of the extent of gas dispersion and (b) the associated superficial liquid velocity in the high temperature gas stirred ladle $(L=0.2 \mathrm{~m}, R=$ $0.15 \mathrm{~m}$ and $W_{o}=180 \mathrm{~m} / \mathrm{s}$ ) [reproduced from Ref. 10)].

system. ${ }^{10)}$ Referring to Fig. 2(a), it is readily seen that the gas injected through the bottom of the ladle becomes considerably dispersed in the reactor vessel. Furthermore, the dispersed phase is predicted to occupy about $40 \%$ of the reactor volume. Consequently, a large proportion of the injected gas is also seen to be dragged along with the radially out flowing liquid at the top of the gas-liquid plume, leading to gas dispersion in the vicinity of the free surface occupying almost $30 \%$ of the bath depth. As a result of these, a markedly different flow pattern is seen in Fig. 2(b), in direct contrast to those characteristic of axisymmetric gas bubble driven systems (e.g., a single recirculating vortex in the bulk liquid. ${ }^{9,13,16)}$ Figure 2(b) clearly reveals that there are at least two or more recirculating vortices in the gas stirred system, one within the main bulk of liquid and the other within the upper gas-liquid dispersion zone. Indeed, the flow pattern shown in Fig. 2(b) appears to be far more complex than those expected under typical axisymmetric ladle refining conditions. The presence of multiple recirculating vortices in the system would, as a consequence, appear to indicate that the rate of transport of the tracer added in the immediate vicinity of the free surface to the bulk liquid and subsequent dispersion, is likely to be different than those one would normally anticipate in gas stirred systems with a single recirculating vortex.

In much contrast to the results of Tukoglu and Farouk (viz., Fig. 2), a typical gas liquid plume and velocity profile deduced experimentally by the present authors and summarised in Ref. 9) is shown in Fig. 3. There, the plume is seen to occupy barely $1 \%$ of the reactor volume and the fluid flow pattern comprises of a single recirculating loop. On the basis of these and considering the fact that the hydrodynamic phenomena at play strongly influence the rate of various transport controlled processes, it should be readily apparent that the two studies $^{9,10)}$ pertain to widely different experimental configurations and bear no comparison. Further, it is not appropriate to conclude purely on the basis of Turkoglu and Farouk's computations that Eq. (4) (an
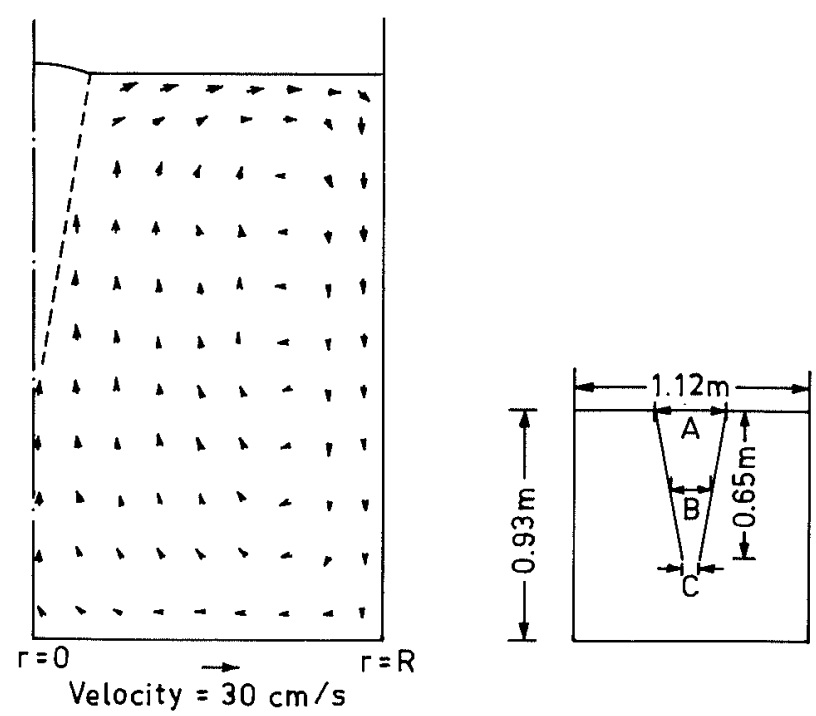

Fig. 3. Experimentally measured geometry of the gas-liquid plume and the associated liquid velocity field in a water model ladle at a gas flow rate of $6.6 \times 10^{-4} \mathrm{~m}^{3} / \mathrm{s}$ [reproduced from Ref. 16)].

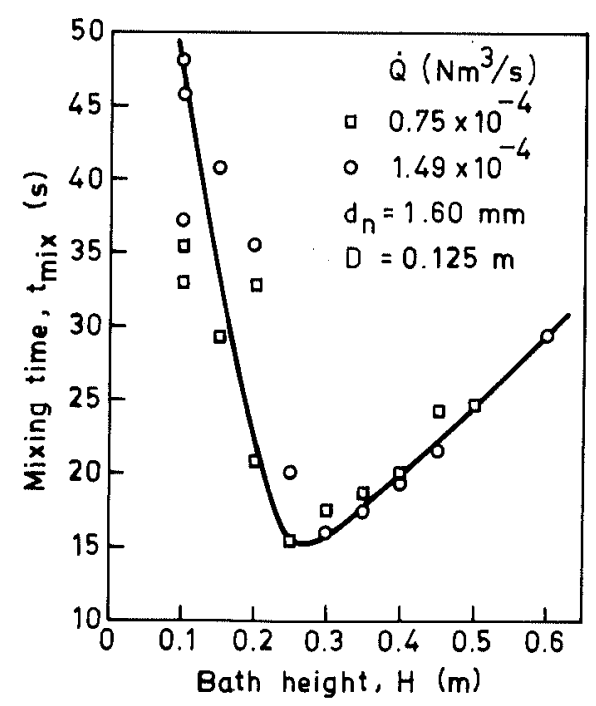

Fig. 4. Experimentally measured mixing times as a function of vessel height at two different gas flow rates [reproduced from Ref. 6)].

expression confirmed experimentally), does not provide realistic estimates of mixing times in gas stirred ladle systems.

Referring back to Fig. 1, it is also seen that beyond a ladle aspect ratio of 1.5 , mixing times increased, for Turkoglu and Farouk, while inferences made on the basis of Eq. (4) indicate quite the opposite. Such increases in mixing times at higher vessel aspect ratios has also been observed by Krishnamurthy et al. ${ }^{6)}$ They, following extensive experimental measurements in a water model of a gas stirred bath, concluded that such increases in mixing time are intricately related to changes in the physical characteristics of the gas-liquid region. Thus at large vessel aspect ratios, the bath is practically transformed into a bubble column. One also encounters similar phenomena at any given aspect ratio once the gas flow rate is increased beyond certain critical values. Under such operating regime, Krishnamurthy et al. ${ }^{6}$ 
observed distinct recirculating loops in the bulk fluid coupled with prolonged mixing times (e.g., see Fig. 4). Furthermore, on the basis of these, it is also to be expected that the vessel aspect ratio beyond which the mixing times increases with the increase in the specific energy input rate, will be a function of the gas flow rates applied. As pointed out by Krishnamurthy et al., explicit references to the presence of several recirculating loops in bubble columns have been well documented in the literature. In view of these, the arguments provided by Turkoglue and Farouk to explain their increases in mixing times beyond a vessel aspect ratio of 1.5 (e.g., that additional energy dissipation by friction of the downwardly flowing fluid against the long vertical wall) appear to have little or no validity at all. Rather, the preceding discussion clearly indicates that the differences between the two set of estimates ${ }^{9,10)}$ illustrated in Fig. 1, is associated with the extrapolation of Eqs. (3) and (4) into a regime of operating conditions which are significantly different than those for which they were originally derived. ${ }^{9)}$ It is, therefore, not surprising that Eq. (4) provides estimates much different than those predicted numerically by Turkoglu and Farouk.

\section{Concluding Remarks}

A recent paper published in this journal by Turkoglu and Farouk on the subject of mixing rates in steelmaking ladles with vertical gas injection has been reviewed and compared with previous work of the present authors. On the basis of such comparisons, and other information available from relevant literature, it is shown that some of the analysis presented by Turkoglu and Farouk, and conclusions drawn are erroneous and misleading. The present work, rationalises the anomalies and shows that the flow regime modelled by Turkoglu and Farouk do not correspond to ladle refinig practice.

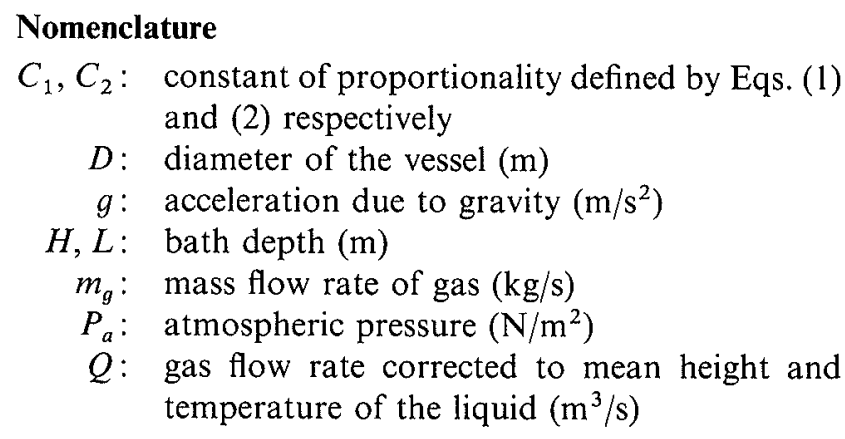

\author{
$R: \quad$ vessel radius $(\mathrm{m})$ \\ $R_{g}:$ gas constant $(\mathrm{J} /(\mathrm{K} \cdot \mathrm{kg}$ mole $))$ \\ $T:$ temperature $(\mathrm{K})$ \\ $W_{B}$ : rate of potential energy (buoyancy work) input \\ (W) \\ $\rho_{L}:$ liquid density $\left(\mathrm{kg} / \mathrm{m}^{3}\right)$ \\ $\beta$ : fractional depth of lance submergence \\ $\tau_{m}: \quad 95 \%$ bulk mixing time (s) \\ $\varepsilon_{m}: \quad$ specific potential energy input rate $(\mathrm{W} / \mathrm{kg})$
}

\section{REFERENCES}

1) S. Asai, T. Okamoto, J. C. He and I. Muchi: Trans. Iron Steel Inst. Jpn., 23 (1983), 43.

2) D. Oymo and R. I. L. Guthrie: Proc., 4th Process Technology Conference, (1984), 45.

3) F. Oeters, H. C. Dromer and J. Kepura: Proc., SCANINJECT III, (1983), Jernkontoret, 7:1.

4) U. P. Sinha and M. J. Macnallan: Metall. Trans., 16B (1985), 850 .

5) K. Nakanishi, T. Fuji and J. Szekely: Ironmaking Steelmaking, 3 (1975), 193.

6) G. G. Krishnamurthy, S. P. Mehrotra and A. Ghosh: Metall. Trans., 19B (1988), 839.

7) J. Szekely, T. Lehner and C. W. Wang: Ironmaking Steelmaking, 7 (1979), 285.

8) M. Salcudean, K. Y. M. Lai and R. I. L. Guthrie: Can. J. Chem. Eng., 63 (1985), 51.

9) D. Mazumdar and R. I. L. Guthrie: Metall. Trans., 17B (1986), 725.

10) H. Turkoglu and B. Farouk: ISIJ Int., 31 (1991), 1371.

11) M. C. Gunton, H. I. Ronsten, D. B. Spalding and D. G. Tatchell: PHOENICS instruction manual, CHAM technical report/75, (1983).

12) B. E. Launder and D. B. Spalding: Computer Methods in Applied Mechanics and Engineering, 3 (1974), 269.

13) Y. Sahai and R. I. L. Guthrie: Metall. Trnas., 13B (1982), 193.

14) M. Sano and K. Mori: Trans. Iron Steel Inst. Jpn., 23 (1983), 171.

15) M. P. Schwarz: ISIJ Int., 9 (1991), 947.

16) D. Mazumdar and R. I. L. Guthrie: Metall. Trans., 16B (1985), 83.

(Received on September 8, 1992; accepted in final form on November 20, 1992)

\section{Dipak MAZUMDAR ${ }^{1)}$ and Roderick I. L. GUTHRIE}

Department of Mining and Metallurgical Engineering, McGill University, Montreal, Canada H3A 2 A7.

1) On leave from The Indian Institute of Technology, Kanpur, India. 